\title{
Rotating flow over an exponentially shrinking sheet with suction
}

\begin{abstract}
The rotating flow and heat transfer over an exponentially shrinking permeable surface is are theoretically (numerically) presented in this paper. It extends the work of previous researchers to the general situations including mass transfer (suction) on the wall and the case of shrinking surface. The analysis is concentrated on the effects of the dimensionless constant suction and rotation parameter on the skin friction coefficients and local heat transfer. It is found that the solutions of the governing ordinary (similarity) differential equations have two branches, upper and lower branch solutions, in a certain range of the governing parameters.
\end{abstract}

Keyword: Heat transfer; Rotating flow; Stretching/shrinking; Suction/injection 